\title{
Some Problems Connected With Rayleigh Distributions
}

\author{
M. M. Siddiqui \\ Contribution from Boulder Laboratories, National Bureau of Standards, Boulder, Colo.
}

(October 19, 1961)

\begin{abstract}
This is an expository paper presenting the following: (1) the origin, and (2) the properties of the Rayleigh distribution; (3) the most efficient estimators of its parameters; (4) a test of the hypothesis that a set of observations is from a Rayleigh distribution; (5) the distribution of the ratio of two independent Rayleigh variates; and (6) the Rayleigh process derived from a normal process.
\end{abstract}

\section{Introduction}

In some problems of physics and engineering, such as the diffusion process, or the distribution of the power (or amplitude) of electromagnetic waves received through a scattering medium, we are concerned with the resultant of many (two- or threedimensional) random vectors. Such problems are treated in probability theory under the heading of "random walk" in one, two, three, or, in general, $k$ dimensions. Here, we will confine our attention to two-dimensional random walk problems which asymptotically give rise to the so-called Rayleigh distribution. Unfortunately, in radiowave propagation literature the name "Rayleigh distribution" is used indiscriminately both for the distribution of power and of amplitude. To distinguish between the two, we will call them Rayleigh power distribution and Rayleigh amplitude distribution, respectively. In statistical literature the distinction is clear: the Rayleigh power is a multiple of $\chi^{2}$ (with 2 degrees of freedom), whereas the Rayleigh amplitude is a multiple of $\chi$. Since the properties of the Rayleigh power distribution are much neater and better suited for statistical estimation and for testing of hypotheses than the properties of the Rayleigh amplitude distribution, we will study only the Ravleigh power distribution. Important characteristics of the amplitude distribution will be obtained from the relationship amplitude $=(\text { power })^{1 / 2}$. The advantages of power over amplitude are roughly the same as those of variance over standard deviation, or of spectral density over its square root. For example, the powers are additive and amplitudes are not; the sum of two independent $\chi^{2}$ variates is again a $\chi^{2}$ variate with added degrees of freedom, but the sum of two $\chi$ variates is not a $\chi$ variate. It is, therefore, suggested that the name "Rayleigh distribution," if used without any other qualification, be reserved for Rayleigh power distribution, and "Rayleigh variate" for a multiple of $\chi^{2}$ with 2 degrees of freedom.

\section{Notation}

Ordinarily the same letter will be used to designate both a random variable and the argument of its probability density or distribution function. Thus we may say : $x$ is a random variable with probability density function $p(x)$ and distribution function $P(x)$. If it becomes necessary to distinguish between the two to avoid confusion or ambiguity, capital letters will be used for the random variables and lower case letters for the real variables. Thus we may say: $X$ is a random variable and $\operatorname{Pr}(x \leq X \leq x+d x)=p(x) d x$.

The function $P$, with one or more arguments, will be used generically for any distribution function, and $p$ for any density function. Thus $P(x)$ and $P(y)$, in general, will not be the same functions. The former denotes the distribution function of $x$ and the latter that of $y$, and the two may be different.

If $x$ and $y$ are random variables, $E x$ will stand for the expected value of $x$, and $E(x \mid y)$ for the conditional expected value of $x$ given the value of $y$. Thus

$$
\begin{aligned}
E x & =\int_{-\infty}^{\infty} x p(x) d x=\int_{-\infty}^{\infty} \int_{-\infty}^{\infty} x p(x, y) d y d x, \\
E(x \mid y) & =\int_{-\infty}^{\infty} x \frac{p(x, y)}{p(y)} d x .
\end{aligned}
$$

It is easy to see that, if $E x<\infty$,

$$
\begin{aligned}
E[E(x \mid y)]=\int_{-\infty}^{\infty} d y p(y) & \int_{-\infty}^{\infty} x \frac{p(x, y)}{p(y)} d x \\
& =\int_{-\infty}^{\infty} \int_{-\infty}^{\infty} x p(x, y) d y d x=E x .
\end{aligned}
$$

The characteristics of a theoretical distribution, i.e., its population parameters, will be designated by Greek letters such as $\mu, \alpha, \sigma$. Their estimates may be denoted by corresponding Latin letters, with or without subscripts or primes. Thus if $\mu$ denotes the 
mean (which is also the median) of a normal distribution, $m$ may denote the sample mean and $m_{1}$ the sample median.

\section{Asymptotic Distribution of the Resultant}

Let $\vec{r}_{i}=\left(r_{i} \cos a_{i}, r_{i} \sin a_{i}\right), i=1,2, \ldots, n$ be independent random coplanar vectors where all values of $a_{i}(i=1,2, \ldots, n)$ between 0 and $2 \pi$ are equally likely. Let $r_{i}$ be a series of positive constants, or else be positive random variables distributed independently of $a_{i}$. Then

$E r_{i} \cos a_{i}=E r_{i} E \cos a_{i}=E r_{i} \frac{1}{2 \pi} \int_{0}^{2 \pi} \cos a_{i} d a_{i}=0$,

$$
\sigma_{i}^{2} \equiv E r_{i}^{2} \cos ^{2} a_{i}=E r_{i}^{2} E \cos ^{2} a_{i}=\frac{1}{2} E r_{i}^{2} .
$$

Similarly,

$E r_{i} \sin a_{i}=0, E r_{i}^{2} \sin ^{2} a_{i}=\frac{1}{2} E r_{i}^{2}, E r_{i}^{2} \sin a_{i} \cos a_{i}=0$,

so that the components of a vector are uncorrelated. This does not mean, however, that they are statistically independent. We note that if $r_{i}$ is a constant $E r_{i}=r_{i}, E r_{i}^{2}=r_{i}^{2}$.

The resultant vector $\vec{r}=(x, y)$ is given by

$$
x=\sum_{i=1}^{n} r_{i} \cos a_{i}, y=\sum_{i=1}^{n} r_{i} \sin a_{i} .
$$

We immediately have

$$
E x=E y=0, \quad \sigma^{2}=E x^{2}=E y^{2}=\sum_{i=1}^{n} \sigma_{i}^{2} .
$$

Applying the Central Limit Theorem for vector variates [Cramér, 1951, pp. 215-216, 285-286], we obtain the following theorem.

Theorem. Suppose that $\rho_{i}^{3}=\operatorname{Er}_{i}^{3}<\infty$ for all $i$, and write

$$
\rho^{3}=\rho_{1}^{3}+\ldots+\rho_{n}^{3} .
$$

If the condition

$$
\lim _{n \rightarrow \infty} \rho / \sigma=0
$$

is satisefid, then, noting that $x$ and $y$ are uncorrelated, we obtain the asymptotic distribution of $(x, y)$ as

$$
d P(x, y)=\frac{1}{2 \pi \sigma^{2}} e^{-\frac{x^{2}+y^{2}}{2 \sigma^{2}}} d x d y, \quad-\infty \leq x, \quad y \leq \infty .
$$

Thus $x / \sigma$ and $y / \sigma$ are asymptotically independent standard normal variates.

We note that if $r_{i}$ have identical distributions and $E r_{1}^{3}<\infty$, or if $r_{i}=r$, a constant for all $i, \rho / \sigma=O\left(n^{-1 / 6}\right)$, which tends to zero as $n \rightarrow \infty$.
If in the equation above we make the transformations

$$
x=r \cos a, \quad y=r \sin a,
$$

the Jacobian of the transformation is $r$, and the distribution of $(r, a)$ is given by

$$
d P(r, a)=\frac{r}{\sigma^{2}} e^{-\frac{r^{2}}{2 \sigma^{2}}} d r \cdot \frac{d a}{2 \pi}, \quad 0 \leq r \leq \infty, \quad 0 \leq a \leq 2 \pi .
$$

Hence the resultant phase, $a$, and the resultant amplitude, $r$, are independently distributed. We will write $\gamma=2 \sigma^{2}$, so that the distribution of $r$ is

$$
d P(r)=\frac{2 r}{\gamma} e^{-\frac{r^{2}}{\gamma}} d r, \quad 0 \leq r \leq \infty .
$$

This distribution is known as Rayleigh amplitude distribution. Making further the transformation

$$
z=r^{2}
$$

we obtain the distribution of $z$, called the Rayleigh power distribution, as

$$
d P(z)=\frac{1}{\gamma} e^{-\frac{z}{\gamma}} d z, \quad 0 \leq z \leq \infty .
$$

We note that $z=x^{2}+y^{2}$ is the sum of two independent normal variates, $x$ and $y$, each with mean zero and variance $\gamma / 2$. Thus $2 z / \gamma$ is a $\chi^{2}$ variate with 2 degrees of freedom [Cramér 1951, p. 233].

In case all $r_{i}=1$ in (3.4), Kluyver [1906] showed that

$$
P(r)=r \int_{0}^{\infty}\left[J_{0}(t)\right]^{n} J_{1}(r t) d t .
$$

If we make the transformation

and observe that

$$
t=2 n^{-1 / 2} y
$$

$$
\lim _{n \rightarrow \infty}\left[J_{0}\left(2 n^{-1 / 2} y\right)\right]^{n}=e^{-y^{2}},
$$

an asymptotic approximation to (3.11) is obtained as

$$
P(r) \cong 1-e^{-\frac{r^{2}}{n}}
$$

This formula is originally due to Rayleigh [1919].

In general, if we start with $k$ dimensional vectors $\vec{r}_{i}=\left(x_{i 1}, \ldots, x_{i k}\right)$, and $\vec{r}=\sum_{i=1}^{n} \vec{r}_{i}=\left(x_{1}, \ldots, x_{k}\right)$, then, under the conditions of the Central Limit Theorem, $x_{1}, \ldots$. ., $x_{k}$ are asymptotically independent normal variates, each with mean zero, and the same variance, $\gamma / 2$. Let $z=x_{1}^{2}+\ldots+x_{k}^{2}$. Then $2 z / \gamma$ is a $\chi^{2}$ variate with $k$ degrees of freedom [Cramér 1951, p. 233]. Thus the asymptotic distribution of $z$ is given by 
$d P(z)=\left[\gamma^{\frac{k}{2}} \Gamma\left(\frac{k}{2}\right)\right]^{-1} e^{-\frac{z}{\gamma} z^{\frac{k}{2}-1}} d z ; \quad 0 \leq z \leq \infty$.

\section{Rayleigh Power Distribution}

\subsection{Distribution Characteristics}

In this section we will study some population characteristics of the Rayleigh power distribution. In the following sections the problems of estimation and of testing of hypotheses concerning this distribution will be considered.

The probability density function of $z$ is

$$
p(z)=\frac{1}{\gamma} e^{-z / \gamma}, \text { if } z \geq 0 ; 0, \text { otherwise, }
$$

which has a maximum at $z=0$ and decreases monotonically as z increases.

The distribution function, $\operatorname{Pr}(Z \leq z)$, is given by

$$
P(z)=\frac{1}{\gamma} \int_{0}^{z} e^{-\frac{x}{\gamma}} d x=1-e^{-\frac{z}{\gamma}}
$$

If $q$ is a number between 0 and 1 , the $100 q$ percentile point, $\zeta_{q}$, is defined by the equation

$$
P\left(\zeta_{q}\right)=q,
$$

which has the solution

$$
\zeta_{q}=-\gamma \ln (1-q) .
$$

In particular the median

$$
\zeta_{.5}=\gamma \ln 2=0.69315 \gamma .
$$

The values of $\zeta_{q} / \gamma$ for $q=0.01,0.05,0.10,0.25,0.50$, $0.75,0.90,0.95,0.99$, are given in table 1 .

TABLE 1. $\zeta_{\mathrm{a}} / \gamma$ for selected $\mathrm{q}$

\begin{tabular}{c|c|c|c|c|c|c|c|c|c}
\hline \hline$q$ & 0.01 & 0.05 & 0.10 & 0.25 & 0.50 & 0.75 & 0.90 & 0.95 & 0.99 \\
\hline$\zeta_{q / \gamma}$ & 0.01005 & 0.05129 & 0.10536 & 0.28768 & 0.69315 & 1.38629 & 2.30259 & 2.99573 & 4.60517 \\
\hline
\end{tabular}

If $k$ is any real number greater than -1

$$
\mu_{k}(z)=E z^{k}=\frac{1}{\gamma} \int_{0}^{\infty} z^{k} e^{-\frac{z}{\gamma}} d z=\gamma^{k} \Gamma(k+1) .
$$

Thus the mean, $\mu_{1}(z)$, and the variance, $\sigma^{2}(z)$, are

$$
\mu_{1}(z)=\gamma, \sigma^{2}(z)=\mu_{2}(z)-\mu_{1}^{2}(z)=\gamma^{2} .
$$

Also the mean and variance of $r=z^{1 / 2}$ are given by

$$
\begin{aligned}
E r & =\mu_{1 / 2}(z)=\gamma^{1 / 2} \Gamma(3 / 2)=1 / 2(\pi \gamma)^{1 / 2}, \\
\sigma^{2}(r) & =\mu_{1}(z)-\mu_{1 / 2}^{2}(z)=\gamma \frac{(4-\pi)}{4} .
\end{aligned}
$$

\subsection{Estimation}

The Rayleigh distribution is completely specified if the parameter $\gamma$ is known. If $z_{1}, z_{2}, \ldots ., z_{N}$ is a sample of $N$ independent observations from this distribution, the likelihood function (the joint probability density of $z_{1}, \ldots, z_{N}$ considered as a function of $\gamma$ ) is given by

$$
L(\gamma)=\frac{1}{\gamma^{N}} e^{-\Sigma z_{i}}=\frac{1}{\gamma^{N}} e^{-\frac{N c}{\gamma}},
$$

where

$$
c=\frac{1}{N} \sum_{i=1}^{N} z_{i}
$$

This shows that $c$ is sufficient for $\gamma$. Since $E c=\gamma$, $c$ is unbiased. We know that if an unbiased sufficient estimator exists, it is automatically the most efficient estimate, also the maximum likelihood estimate. The variance of $c$ is, of course,

$$
\operatorname{var} c=\frac{\gamma^{2}}{N}
$$

If $t$ is any other unbiased estimate of $\gamma$, then var $c \leq \operatorname{var} t$.

Since $2 z_{i} / \gamma$ is a $\chi^{2}$ variate with 2 degrees of freedom, and $z_{1}, \ldots, z_{N}$ are independent

$$
2 N c / \gamma=2 \sum_{i=1}^{N} z_{i} / \gamma
$$

is a $\chi^{2}$ variate with $2 N$ degrees of freedom. Thus the distribution of $c$ is

$$
d P(c)=\frac{N^{N}}{\gamma^{N} \Gamma(N)} e^{-\frac{N c}{\gamma}} c^{N-1} d c \text {, if } c \geq 0 ;=0 \text {, otherwise. }
$$

To set up confidence limits on $\gamma$ with a confidence coefficient $1-\alpha$, we determine from $\chi^{2}$ tables two numbers $\chi_{1}^{2}$ and $\chi_{2}^{2}$, corresponding to $2 N$ degrees of freedom, such that

Then

$$
\operatorname{Pr}\left(\chi^{2} \leq \chi_{1}^{2}\right)=\alpha / 2, \operatorname{Pr}\left(\chi^{2} \leq \chi_{2}^{2}\right)=1-\alpha / 2 .
$$

$$
\chi_{1}^{2} \leq 2 N c / \gamma \leq \chi_{2}^{2},
$$

has probability $1-\alpha$ and

$$
\frac{2 N c}{\chi_{2}^{2}} \leq \gamma \leq \frac{2 N c}{\chi_{1}^{2}},
$$

will be $100(1-\alpha)$ percent confidence limits for $\gamma$.

Example 1. Let $N=3,1-\alpha=0.98$. From $\chi^{2}$ tables we find, for 6 degrees of freedom, $\mathrm{Pr}$ $\left(\chi^{2} \leq 0.872\right)=0.01, \operatorname{Pr}\left(\chi^{2} \leq 16.812\right)=0.99$. Hence

$$
\frac{6}{16.812} c \leq \gamma \leq \frac{6}{0.872} c
$$

or

$$
0.357 c \leq \gamma \leq 6.88 c
$$


are 98 percent confidence limits for $\gamma$. Similarly

$$
0.476 c \leq \gamma \leq 3.67 c
$$

are 90 percent confidence limits.

If $N>15$ so that the number of degrees of freedom $f=2 N>30$,

$$
\sqrt{2 \chi^{2}}-\sqrt{2 f-1}=\sqrt{\frac{4 N c}{\gamma}}-\sqrt{4 N-1}
$$

is approximately a standard normal variate [Cramér, 1951, p. 251]. If $x$ is a number such that

$$
\frac{1}{\sqrt{2 \pi}} \int_{-x}^{x} e^{-\frac{y^{2}}{2}} d y=1-\alpha,
$$

then

$$
\frac{4 N c}{(\sqrt{4 N-1}+x)^{2}} \leq \gamma \leq \frac{4 N c}{(\sqrt{4 N-1}-x)^{2}}
$$

are $100(1-\alpha)$ percent confidence limits for $\gamma$. When $N$ is large, say $N>100,(4.12)$ is approximated by

$$
\frac{\sqrt{N} c}{\sqrt{N}+x} \leq \gamma \leq \frac{\sqrt{N} c}{\sqrt{N}-x} .
$$

Example 2. Let $N=100,1-\alpha=0.95$. We find $x=1.96$. From (4.12)

$$
0.831 c \leq \gamma \leq 1.23 c .
$$

If, on the other hand, we use (4.13), we get

$$
0.836 c \leq \gamma \leq 1.24 c \text {. }
$$

Since $c$ is the sufficient statistic for the Rayleigh distribution, if it is known that the sample has come from a Rayleigh distribution, we simply evaluate $c_{\mathbf{a}}$ and estimate the entire distribution by

$$
\hat{P}(z)=1-e^{-\frac{z}{c}}, 0 \leq z \leq \infty .
$$

The usual practice of estimating the population percentiles by the sample percentiles is utterly unjustified. The distribution percentile, $\zeta_{q}$, should be estimated by

$$
z_{q}=-c \ln (1-q),
$$

which is an unbiased and minimum variance estimator of $\zeta_{q}$. Thus, for example, the median, $\zeta_{.5}$, will be estimated by

$$
z_{.5}=c \ln 2=0.69315 c,
$$

which has the variance

$$
\operatorname{var} z_{.5}=(\ln 2)^{2} \operatorname{var} c=\frac{0.480 \gamma^{2}}{N} .
$$

On the other hand, the asymptotic variance of the sample median, $z_{.5}^{\prime}$, is given by

$$
\operatorname{var} z_{.5}^{\prime} \cong \frac{1}{4 N p^{2}\left(\zeta_{.5}\right)}=\frac{\gamma^{2}}{N} .
$$

Thus

$$
\frac{\operatorname{var} z_{.5}}{\operatorname{var} z_{.5}^{\prime}} \cong 0.480
$$

i.e., the sample median is only 48 percent as efficient as $z .5$.

To take another example: the first decile, $\zeta .1$, is most efficiently estimated by

$$
z_{.1}=-c \ln (.90)=0.10536 c,
$$

which is unbiased and has variance

$$
\operatorname{var} z_{.1}=0.0111 \frac{\gamma^{2}}{N} \text {. }
$$

The sample 1st decile, $z_{.1}^{\prime}$ is biased and has asymptotic variance

$$
\operatorname{var} z_{.1}^{\prime}=\frac{0.1 \times 0.9}{N p^{2}\left(\zeta_{.1}\right)}=\frac{\gamma^{2}}{9 N}
$$

so that

$$
\frac{\operatorname{var} z_{.1}}{\operatorname{var} z_{.1}^{\prime}} \cong 9 \times 0.0111 \cong 0.1,
$$

i.e., the efficiency of $z^{\prime}{ }_{1}$ is only 10 percent.

\subsubsection{Estimation of the Amplitude Characteristics}

We know that if sufficient statistics are available for a distribution, its characteristics are most efficiently estimated in terms of these statistics. Thus the characteristics of the amplitude distribution are estimated in terms of $c$.

For example to estimate the mean amplitude, $(\pi \gamma)^{1 / 2} / 2$, we need only to find a function of $c$ which is an unbiased estimator of this quantity.

Now

$$
\begin{aligned}
E c^{\frac{1}{2}} & =E(\gamma+c-\gamma)^{\frac{1}{2}} \\
& =\gamma^{\frac{1}{2}} E\left[1+\frac{1}{2} \frac{c-\gamma}{\gamma}-\frac{1}{8} \frac{(c-\gamma)^{2}}{\gamma^{2}}+\ldots\right] \\
& \simeq \gamma^{\frac{1}{2}}\left[1-\frac{1}{8 N}\right] .
\end{aligned}
$$

Thus, to the order $N^{-1},[1+1 /(8 N)] c^{1 / 2}$ is an unbiased estimate of $\gamma^{1 / 2}$. The mean of $r$ is then estimated by

$$
m=\frac{1}{2}\left(1+\frac{1}{8 N}\right) \sqrt{\pi c} .
$$


Since

$$
\begin{aligned}
\operatorname{var} c^{\frac{1}{2}} & =E c-\left(E c^{\frac{1}{2}}\right)^{2} \\
& \cong \gamma-\gamma\left(1-\frac{1}{8 N}\right)^{2} \cong \frac{\gamma}{4 N}, \\
\operatorname{var} m & \cong \frac{\pi \gamma}{16 N} .
\end{aligned}
$$

On the other hand, let $r_{i}=z_{i}^{\frac{1}{2}}$, and

$$
\bar{r}=\frac{1}{N} \sum_{i=1}^{N} r_{i} .
$$

We have

$$
\operatorname{var} \bar{r}=\frac{\operatorname{var} r_{i}}{N}=\frac{\gamma(4-\pi)}{4 N}
$$

Thus

$$
\frac{\operatorname{var} m}{\operatorname{var} \bar{r}}=\frac{\pi}{4(4-\pi)} \simeq 0.91
$$

i.e., the sample mean, $\bar{r}$, is only 91 percent as efficient as the unbiased estimator based on the sufficient statistic $c$.

Similar considerations apply to estimating other characteristics of the amplitude distribution, and hence there is no need for us to go into details.

\subsubsection{Estimation When the Data Are Truncated}

Sometimes, due to the limitations of the recording instrument, observations above a certain level $x$ are not recorded. However, the prior information is there that the unrecorded observations will be greater than $x$, and the recorded ones will be less than $x$. This prior information together with the actual values of the recorded observations should be utilized to obtain the most efficient estimator of $\gamma$.

In a sample of $N$ independent observations let $N-k$ values exceed $x$, and let the remaining $k$ observations be $z_{1}, z_{2}, \ldots, z_{k}$. Obviously, $k$ itself is a random variable with possible values $0,1,2, \ldots$, $N$. Also the probability density function of $z_{i}$ is now conditioned upon the knowledge that $0 \leq z_{i} \leq x$.

The conditional probability density function is

$$
p(z \mid 0 \leq z \leq x)=\frac{1}{\gamma p} e^{-z / \gamma}, 0 \leq z \leq x,
$$

where $p=1-e^{-\frac{x}{\gamma}}$. The probability distribution of $k$ is

$$
\operatorname{Pr}(K=k)=\left(\begin{array}{c}
N \\
k
\end{array}\right) p^{k}(1-p)^{N-k}, k=0,1, \ldots, N .
$$

Hence the likelihood function is

$L(\gamma)=\left\{\begin{array}{l}e^{\frac{-N x}{\gamma}}, \text { if } k=0, \\ \left(\begin{array}{l}N \\ k\end{array}\right) \frac{1}{\gamma^{k}} \exp \left[-\frac{1}{\gamma}\left\{(N-k) x+\sum_{i=1}^{k} z_{i}\right\}\right], \\ 0 \leq z_{1}, \ldots, z_{k} \leq x, k=1,2, \ldots, N .\end{array}\right.$
Obviously, when $k=0$ no point estimate of $\gamma$ is possible, since the only information available is that all observations are greater than $x$. In this case, which has probability $e^{-\frac{N x}{\gamma}}$, we can only infer that $x<\gamma<\infty$. When $k \neq 0$, the maximum likelihood estimate of $\gamma$ is

$$
c_{1}=\frac{(N-k) x+\sum_{i=1}^{k} z_{i}}{k},
$$

The properties of $c_{1}$ are not as easy to establish as those of $c$ in section 4.2. Using the well known properties of the maximum likelihood estimators, however, we can say that for large $N, c_{1}$ will be normally distributed with mean $E c_{1}$, and variance, var $c_{1}$, where

$$
\begin{aligned}
E c_{1} & \simeq \gamma+\frac{x(1-p)}{N p^{2}}, \\
\operatorname{var} c_{1} & \simeq \frac{2 \gamma^{2}}{N p}-\frac{(4 p-3) x^{2}}{N p^{3}}, p=1-e^{-\frac{x}{\gamma}} .
\end{aligned}
$$

\subsection{Is the Variate Rayleigh Distributed?}

Let $z_{1}, z_{2}, \ldots, z_{N}$ be independent observations on a non-negative variate $z$. We wish to test whether $z$ is Rayleigh distributed. Proceed in the following manner.

Choose an integer $m$, not too small (at least 5), such that $N / m$ is greater than 5 , preferably greater than 10. Calculate $c=\sum_{i=1}^{N} z_{i} / N$. Determine numbers $x_{1}, \ldots, x_{m-1}$, such that

$$
\hat{P}\left(x_{i}\right)=1-e^{-\frac{x_{i}}{c}}=\frac{i}{m}, i=1,2, \ldots, m-1 \text {, }
$$

i.e.,

$$
x_{i}=c \ln \left(\frac{m}{m-i}\right), i=1,2, \ldots, m-1 .
$$

The range $(0, \infty)$ of $z$ is then divided into $m$ nonoverlapping intervals $I_{1}=\left(0, x_{1}\right), I_{2}=\left(x_{1}, x_{2}\right), \ldots . ., I_{m}=$ $\left(x_{m-1}, \infty\right)$, such that the expected number of observations, under the hypothesis $\hat{P}(z)$, in each interval is $N / m$.

Let the number of actual observations falling in these intervals be $f_{1}, f_{2}, \ldots, f_{m}$. Then

$$
\chi_{0}^{2}=\sum_{i=1}^{m} \frac{\left(f_{i}-N / m\right)^{2}}{N / m}=\frac{m}{N} \sum_{i=1}^{m} f_{i}^{2}-N
$$

is approximately a $\chi^{2}$ variate with $m-2$ degrees of freedom [Cramér, 1951, pp. 424-426]. Pre-assign some critical probability level $\alpha(=0.05$, or 0.01 , say $)$ and let $\chi_{\alpha}^{2}$ be the number such that

$$
\operatorname{Pr}\left(\chi^{2} \geq \chi_{\alpha}^{2}\right)=\alpha .
$$

If the observed value $\chi_{0}^{2}<\chi_{\alpha}^{2}$, accept the hypothesis that $z$ is a Rayleigh variate, and estimate its dis- 
tribution function by $\hat{P}(z)$. If $\chi_{0}^{2} \geq \chi_{\alpha}^{2}$, reject the hypothesis of Rayleigh distribution.

If the sample is truncated, i.e., if the maximum value which could be recorded is $x$, we estimate $\gamma$ by $c_{1}=\left[(N-k) x+\sum_{i=1}^{k} z_{i}\right] / k$, where $N-k$ values are greater than $x$ and unrecorded. We proceed as above, changing the last interval (or intervals), if necessary, to read $(x, \infty)$ and determining the expected number of observations in this interval by $N e^{-x / c_{1}}$; and calculating $\chi_{0}^{2}=\sum \frac{\left(f_{o}-f_{e}\right)^{2}}{f_{e}} ; f_{o}=o b-$ served, $f_{e}=$ expected frequency.

Example 3. The following is a systematic sample of 80 observations (read at 5 sec intervals) of received field intensity in (microvolts) ${ }^{2}$. The following are the transmission parameters:

Frequency:
Transmitter
Angle:
Path length :
Antennas:

Loss :

Time:

Observed values of received power in $(\mu \mathrm{v})^{2}(\mathrm{read}$ left to right):

$0.20,0.71,0.06,0.05,0.76,0.32,0.96,0.63,0.09$, $0.18,0.25,0.45,0.26,0.10,0.95,0.01,0.50,1.26$, $1.99,0.32,0.51,0.01,0.16,0.56,3.16,1.27,2.24$, $1.00,0.81,1.29,0.28,0.21,0.35,0.20,0.39,0.89$, $1.24,0.08,0.98,1.01,0.49,0.90,1.90,1,42,1.56$, $1.32,1.20,1.59,2.40,2.24,0.80,0.56,1.45,0.18$, $0.02,0.28,0.81,0.18,1.31,0.64,1.95,0.48,0.55$, $0.44,0.28,0.07,0.71,0.48,0.40,0.06,0.79,1.01$, $0.51,0.70,0.14,0.16,0.01,0.06,0.03,0.01$.

We have $N=80, c=0.71$. Take $m=8, \alpha=0.05$. For 6 degrees of freedom the critical value of $\chi^{2}$ is 12.592 .

Calculate $x_{1}=-0.71 \ln \frac{8-i}{8}, i=1,2, \ldots . \quad$, 7 . The resulting class intervals and the observed frequencies are as follows.

\begin{tabular}{r|r}
\hline \hline & \\
Class intervals & $f_{o}$ \\
\hline & \\
\hline $0 \quad-0.095$ & 13 \\
$0.095-.204$ & 9 \\
$.204-.334$ & 8 \\
$.334-.492$ & 8 \\
$.492-.696$ & 8 \\
$.696-.984$ & 13 \\
$.984-1.476$ & 12 \\
$1.476-\infty$ & 9 \\
\hline
\end{tabular}

The expected frequency in each interval is 10 . We have

$$
\chi_{0}^{2}=\frac{8}{80} \sum f^{2}-80=3.6<12.592 .
$$

The data are consistent with the hypothesis that $z$ is Rayleigh distributed. In fact, the probability that such a sample or worse, as measured by $\chi^{2}$, comes from a Rayleigh distribution is more than 70 percent. The entire distribution is estimated by

$$
\hat{P}(z)=1-e^{-\frac{z}{0.71}}, 0 \leq z \leq \infty .
$$

\subsection{Two Independent Samples}

Sometimes we wish to test the hypothesis that two samples, which are known to be from. Rayleigh distributions, are from the same distribution, i.e., $\gamma_{1}=\gamma_{2}$. For example, to test the assumption of stationarity of a Rayleigh process, we may take two samples from the record (sufficiently far apart in time to insure independence), and test the hypothesis that $\gamma_{1}=\gamma_{2}$. Or, we may be recording a signal, not continuously, but after breaks in between recordings, and may wish to test whether the characteristics of the received signal have changed from record to record.

Let it be known that two independent samples, $z_{1}, z_{2}, \ldots . z_{N}$, and $z_{1}^{\prime}, z_{2}^{\prime}, \ldots ., z^{\prime}{ }_{M}$, are from Rayleigh distributions. To test the hypothesis that $\gamma_{1}=\gamma_{2}$, it is sufficient to note that

$$
F(2 N, 2 M)=\frac{\sum_{i=1}^{N} z_{i}}{2 N} \cdot \frac{2 M}{\sum_{i=1}^{M} z_{i}^{\prime}}=\frac{c_{1}}{c_{2}}
$$

is a Fisher-Snedecor $F$ variate with the indicated degrees of freedom. Here, $c_{1}$ and $c_{2}$ denote the sample means of the first and second sample respectively. We assume that $c_{1} \geq c_{2}$; if not we simply invert the ratio and interchange the degrees of freedom. Pre-assign a significance level $\alpha$, and test for the significance of the calculated $F$.

Example 4. Consider the data in example 3. Let us test the hypothesis that the first 25 observations are from the same Rayleigh distribution as the last 25 observations. Let the significance level be taken as 0.05 . From $\mathrm{F}$ tables the 5 percent significant value, for 50 and 50 degrees of freedom, is 1.60. Denoting by $c_{1}$ and $c_{2}$ the mean values of the first 25 and the last 25 observations, respectively, we have

$$
c_{1}=0.578, \quad c_{2}=0.482 \text {. }
$$

Hence, the calculated value of $F$ is

$$
F_{c}=\frac{578}{482}=1.20
$$

which is less than 1.60. Hence we accept the hypothesis that $\gamma_{1}=\gamma_{2}$. 


\section{Distribution of the Ratio of Two Rayleigh Variates}

Many problems in radio engineering require the knowledge of the distribution of the ratio of two independent Rayleigh variates, such as the signal power and the noise power, or the powers of two interfering signals.

Let $z_{1}$ and $z_{2}$ be two independent Rayleigh variates with parameters $\gamma_{1}$ and $\gamma_{2}$. Let $u=z_{1} / z_{2}$, and $\epsilon=\gamma_{1} / \gamma_{2}$. Since $2 z_{1} / \gamma_{1}$ and $2 z_{2} / \gamma_{2}$ are independent $\chi^{2}$ variates each with 2 degrees of freedom,

$$
u_{1}=\frac{z_{1} / \gamma_{1}}{z_{2} / \gamma_{2}}=\frac{z_{1}}{\epsilon z_{2}}
$$

is a Fisher-Snedecor $F$ variate with 2 and 2 degrees of freedom [Cramér, 1951, pp. 241-242]. Hence the distribution of $u=\epsilon U_{1}$ is given by

$$
\begin{gathered}
d P(u)=\frac{\epsilon d u}{(\epsilon+u)^{2}}, \quad 0 \leq u \leq \infty ; \\
1-P(u)=\operatorname{Pr}\left(z_{1} / z_{2} \geq u\right)=\frac{\epsilon}{\epsilon+u} .
\end{gathered}
$$

Example 5. Let the average signal power be $20 \mathrm{db}$ above the average noise power so that $\epsilon=100$. Let satisfactory service require signal power $10 \mathrm{db}$ above the noise power. To calculate the probability of satisfactory service we set $\epsilon=100, u=10$ in (5.2) and obtain $\operatorname{Pr}\left(z_{1} / z_{2} \geq 10\right)=0.91$

The distribution of $u$ involves only one parameter $\epsilon=\gamma_{1} / \gamma_{2}$, which is the median of the distribution. We note that the mean, variance, and higher moments of $u$ do not exist.

Let $u_{1}, \ldots, u_{N}$ be independent observations on $u$. The logarithm of the likelihood function is

$$
\ln L(\epsilon)=N \ln \epsilon-2 \sum_{i=1}^{N} \ln \left(\epsilon+u_{i}\right),
$$

which shows that no sufficient estimator exists for $\epsilon$. The maximum likelihood estimator, $e$, of $\epsilon$ is the solution of the equation

$$
\left.\frac{\partial \ln L}{\partial \epsilon}\right|_{\epsilon=e}=0
$$

i.e., of

$$
\frac{N}{e}-\sum_{i=1}^{N} \frac{2}{e+u_{i}}=0
$$

This equation is quite difficult to solve if $N>3$. We, therefore, find some less efficient but easily available estimator. The Cramér-Rao greatest lower bound for the variance of any unbiased estimator is found to be

$$
v=\left[E\left(\frac{\partial \ln L}{\partial \epsilon}\right)^{2}\right]^{-1}=\frac{3 \epsilon^{2}}{N},
$$

which is also the asymptotic variance of $e$. The asymptotic variance of the sample median, $u_{.5}^{\prime}$, is given by

$$
\operatorname{var} u_{.5}^{\prime} \cong \frac{1}{4 N p^{2}(\epsilon)}=\frac{4 \epsilon^{2}}{N},
$$

so that the efficiency of the sample median is 75 percent. Thus, for a quick estimate of $\epsilon$, the median may be used. In any case the sample mean should not be taken as an estimate of $\epsilon$.

\section{A Rayleigh Process Derived From Normal Process}

Referring back to the theoretical framework of section 3, we assume now that the resultant vector $\vec{r}$ is a function of time, i.e., $\vec{r}(t)$ is a random vector process. The components $(x, y)$ will now be written $(x(t), y(t))$ and it will be assumed that $x(t)$ and $y(t)$ are independent stationary normal processes with the same variance $\sigma^{2}$ and the same autocorrelation function $\rho(s)$. That is to say, for all $t$ and $s$

$$
\begin{aligned}
& E x^{2}(t)=E y^{2}(t)=\sigma^{2}, \\
& E x(t) x(t+s)=E y(t) y(t+s)=\sigma^{2} \rho(s) .
\end{aligned}
$$

Set

$$
z(t)=x^{2}(t)+y^{2}(t), \quad \gamma=2 \sigma^{2} .
$$

Then, for all $t$ and $s$,

$$
\begin{aligned}
& E z(t)=\gamma, \\
& E z(t) z(t+s)=\gamma^{2}\left(1+\rho^{2}(s)\right) .
\end{aligned}
$$

Writing $\alpha(s)$ for the autocorrelation of the $z(t)$ process, we have

$$
\alpha(s)=\frac{\operatorname{cov}(z(t), z(t+s))}{\gamma^{2}}=\rho^{2}(s) .
$$

The process is completely determined if $\gamma$ and $\alpha(s)=\rho^{2}(s)$ are known.

As before we use the sample mean now given by

$$
c=\frac{1}{T} \int_{0}^{T} z(t) d t,
$$

to estimate $\gamma . \quad c$ is unbiased and has variance

$$
\operatorname{var} c=\frac{2 \gamma^{2}}{T} \int_{0}^{T}\left(1-\frac{s}{T}\right) \alpha(s) d s .
$$

We note that in the case of a discrete sample $z(1), z(2), \ldots ., z(N),(6.6)$ will read

$$
\operatorname{var} c=\frac{\gamma^{2}}{N}+\frac{2 \gamma^{2}}{N} \sum_{s=1}^{N-1}\left(1-\frac{s}{N}\right) \alpha(s) .
$$


The exact distribution of $c$ is unknown. However, let $N^{\prime}$ be defined by the equation

$$
\operatorname{var} c=\gamma^{2} / N^{\prime} \text {, i.e., } N^{\prime}=\gamma^{2} / \operatorname{var} c,
$$

where var $c$ is given by $(6.6)$ or $\left(6.6^{\prime}\right) . \quad N^{\prime}$ will be called equivalent random sample size for estimating $\gamma$. The results of sections 4.2-4.4, then, will approximately hold with $N$ and $M$ replaced by equivalent random sample sizes $N^{\prime}$ and $M^{\prime}$. $N^{\prime}$, in general, will not be an integer, but this presents no difficulty either in theory or in calculations. If $\left[2 N^{\prime}\right]$ is the largest integer in $2 N^{\prime}$, we will simply interpolate between the percentiles of $\chi^{2}$ for $\left[2 N^{\prime}\right]$ and $\left[2 N^{\prime}\right]+1$ degrees of freedom to obtain the percentile points of $2 N^{\prime} c / \gamma$. Similarly, for $F\left(2 N^{\prime}, 2 M^{\prime}\right)$ of section 4.4 .

Example 6. Let $\alpha(s)=\alpha(-s)=e^{-\mu s}, s \geq 0, \mu>0$. Also, let $\rho=e^{-\mu}$, so that $\alpha(s)=\rho^{s}$. From $(6.6)$

$$
\operatorname{var} c=\frac{2 \gamma^{2}}{\mu T}\left[1-\frac{1-e^{-\mu T}}{\mu T}\right] \simeq \frac{2 \gamma^{2}}{\mu T}
$$

if $\mu T$ is large. Hence, the equivalent random sample size $N^{\prime} \cong \mu T / 2$.

In case of a discrete sample, we obtain from. $\left(6.6^{\prime}\right)$

$$
\operatorname{var} c \cong \frac{\gamma^{2}}{N}\left(\frac{1+\rho}{1-\rho}\right)
$$

so that $N^{\prime} \cong N(1-\rho) /(1+\rho)$.

For example, if $e^{-\mu}=\rho=0.5, \quad \mu=0.69315,2 N^{\prime}=$ $0.69315 T$ in the continuous sample case, and $2 N^{\prime}=$ $2 N / 3$ in the discrete sample case.
Let

$$
C(s)=\frac{1}{T-s} \int_{0}^{T-s} z(t) z(t+s) d t .
$$

Then

$$
a(s)=\frac{C(s)}{c^{2}}-1
$$

is a consistent estimate of $\alpha(s)$.

From the joint distribution of $z(t)$ and $z(t+s)$, obtained from Siddiqui's [1961] equations (5.8) and (5.9) by setting $n=2$, we also find the following:

$$
E(z(t+s) \mid z(t))=\alpha(s) z(t)+\gamma(1-\alpha(s)),
$$

so that the regression of $z(t+s)$ on $z(t)$ is linear. However,

$\operatorname{var}(z(t+s) \mid z(t))=\gamma^{2}(1-\alpha(s))^{2}+2 \gamma \alpha(s)(1-\alpha(s)) z(t)$,

is not independent of $z(t)$.

\section{References}

Cramér, H., Mathematical methods of statistics (Princeton University Press, Princeton, 1951).

Kluyver, J. C., A local probability problem, Proc. Nederl. Akad. Wetensch. 8, 341-350 (1906).

Rayleigh, J. W. Strutt, Lord, On the problem of random vibrations and of random flights in one, two, and three dimensions, Phil. Mag. 3\%, 321-347 (1919).

Siddiqui, M. M., Some properties of the empirical distribution function of a random process, J. Research NBS 65B (Math. and Math. Phys.), No. 2, 117-127 (April-June 1961).

(Paper 66D2-184) 